\title{
Formation de coke à partir de propène sur silicalite
}

\author{
P. Magnoux ${ }^{1}$, G. Joly ${ }^{1}$, S. Brimaud ${ }^{1}$ et K. Ragil ${ }^{2}$ \\ 1 UMR 6503, Laboratoire de catalyse en chimie organique, 40, avenue du Recteur-Pineau, 86022 Poitiers Cedex - France \\ 2 Institut français du pétrole, 1 et 4, avenue de Bois-Préau, 92852 Rueil-M almaison Cedex - France \\ e-mail : patrick.magnoux@univ-poitiers.fr - guy.joly@univ-poitiers.fr - karine.ragil@ifp.fr
}

Résumé - Sur silicalite et zéolithe MFI acide, l'adsorption du propène a été suivie en microbalance sur une gamme de température variant de 25 à $450^{\circ} \mathrm{C}$ et pour une pression d'alcène de $97 \mathrm{kPa}$. L'analyse de la phase irréversiblement adsorbée (coke) montre que, malgré la faible acidité de la silicalite, dès $25^{\circ} \mathrm{C}$ le propène subit des réactions d'oligomérisation. La quantité maximale de coke est obtenue entre 100 et $150^{\circ} \mathrm{C}$. Pour ces températures, des oligomères avec un nombre de carbone non multiple de trois sont présents dans les pores de la zéolithe, des réactions d'oligomérisation et de craquage sont responsables de la formation de ces composés. Dans ces conditions, on peut estimer qu'environ $10 \%$ de coke entraîne le complet blockage des pores de la silicalite. À plus haute température, des composés aromatiques et polyaromatiques sont formés par réactions successives (oligomérisation, craquage, cyclisation, transfert d'hydrogène). La présence des composés polyaromatiques est responsable de la forte désactivation de l'adsorbant par un rapide bloquage de pores. Sur zéolithe acide $(\mathrm{Si} / \mathrm{Al}=40)$, les réactions de formation de coke sont plus rapides mais les molécules formées sont similaires à celles identifiées dans les pores de la silicalite.

\footnotetext{
Abstract - Coke Formation from Propene over Silicalite Zeolite - The formation of carbonaceous compounds (coke) from propene on silicalite and their effects on the capacity for nitrogen adsorption were investigated in a microbalance system. The operating conditions of coking were as follows: $25-450^{\circ} \mathrm{C}$, pressure of alkene of $97 \mathrm{kPa}$. At ambient temperature $\left(25^{\circ} \mathrm{C}\right)$ silicalite, despite its low acidity, is able to produce oligomers from propene in its pores. For $100-150^{\circ} \mathrm{C}$, reactions of oligomerization/ cracking occur to produce oligomers with a number of carbons different from three and pore blockage occurs for about $10 \%$ of coke. At high temperature $\left(350-450^{\circ} \mathrm{C}\right)$, aromatics and polyaromatics were formed and these coke molecules (most likely the polyaromatic ones) rapidly blocked the access of adsorbates to the zeolite pores. Over more acidic MFI zeolite $(\mathrm{Si} / \mathrm{Al}=40)$, coking rate was more rapid but the nature of coke compounds was similar.
} 


\section{IN TRO DUCTION}

L'isomérisation des $n$-alcanes connaît un nouvel intérêt depuis que la législation a supprimé l'ajout de plomb tétraéthyle dans les essences. Cette réaction permet, en partant de $n$-paraffines de faible indice d'octane, d'obtenir des isoparaffines présentant un indice d'octane beaucoup plus élevé [1]. De plus, dans le cadre d'un durcissement accru de la teneur maximale admissible en composés aromatiques dans les essences, la valorisation des coupes $\mathrm{C}_{7}-\mathrm{C}_{10}$ de distillation directe est rendue nécessaire. Dans cette optique, la production d'isomères multibranchés à haut indice d'octane [1] à partir des heptanes et octanes apparaît comme une voie prometteuse. Ceci justifie pleinement la recherche de systèmes catalytiques performants en isomérisation des coupes $\mathrm{C}_{6}-\mathrm{C}_{8}$ ainsi que la recherche de procédés permettant de recycler sélectivement à l'isomérisation les composés de faible indice d'octane que sont les paraffines linéaires et monobranchées. Le recyclage de ces alcanes vers l'isomérisation implique la séparation préalable des isomères dibranchés.

Dans le cas de mélanges $n /$ isoC $_{5}$, l'utilisation de tamis moléculaire, comme la zéolithe 5A, permet la séparation sélective des isoalcanes (procédé IPSORB développé par l'IFP [2]). La technique de séparation par tamis moléculaire repose sur la faculté de la zéolithe utilisée d'adsorber sélectivement dans ses pores les $n$-paraffines du fait de leurs diamètres cinétiques et de ne pas retenir les isoparaffines à cause de leurs diamètres cinétiques légèrement plus grands. L'étape d'adsorption est suivie d'une étape de désorption pour la récupération des paraffines normales. En ce qui concerne la séparation des alcanes dibranchés dans un mélange résultant de l'isomérisation de la coupe $\mathrm{C}_{6}-\mathrm{C}_{8}$, un tamis moléculaire différent de la zéolithe $5 \mathrm{~A}$ doit être considéré et la silicalite (MFI purement silicique) peut être envisagée pour cette séparation [3-6].

Toutefois, comme dans tout procédé utilisant des zéolithes, un problème majeur, qui intervient au cours de la séparation, concerne la désactivation des tamis moléculaires par perte de capacité d'adsorption. Cette désactivation peut avoir comme origine l'adsorption et la rétention d'alcanes branchés dans les pores de la zéolithe [7, 8] et/ou la formation et la rétention de coke dans les pores du tamis moléculaire. Il a d'ailleurs été montré, au cours de la séparation des $n /$ isoC $_{5}$, que le dépôt carboné formé sur la zéolithe $5 \mathrm{~A}$ pouvait résulter de la transformation d'alcènes [9-13] présents à l'état de trace dans les charges.

À partir de ces connaissances, l'objectif de ce travail a donc été d'évaluer les tendances au cokage d'une silicalite donnée. Pour cela, l'agent cokant utilisé a été le propène, la formation de coke a été étudiée en fonction du temps pour diverses températures $\left(25\right.$ à $\left.450^{\circ} \mathrm{C}\right)$ sur une silicalite de rapport $\mathrm{Si} / \mathrm{Al}=500$. Les cinétiques de formation de coke ont été comparées avec celles obtenues à partir d'une zéolithe MFI acide $(\mathrm{Si} / \mathrm{Al}=40)$. De plus, la nature des composés formés et retenus dans la porosité des deux zéolithes (coke) a été déterminée et comparée sur les deux solides.

\section{PARTIE EXPÉRIMENTALE}

\subsection{Zéolithes}

La zéolithe HMFI(40) $\left(\mathrm{Na}_{0,04} \mathrm{H}_{2,30} \mathrm{Al}_{2,34} \mathrm{Si}_{93,66} \mathrm{O}_{192}\right)$ et la silicalite $\operatorname{HMFI}(500)\left(\mathrm{H}_{0,19} \mathrm{Al}_{0,19} \mathrm{Si}_{95,81} \mathrm{O}_{192}\right)$ utilisées dans cette étude sont des zéolithes commerciales et nous ont été fournies par l'Institut français du pétrole. L'analyse par microscopie électronique montre sur HMFI(40) la présence de cristaux d'environ $1 \mu \mathrm{m}$ et sur HMFI(500) la présence d'agrégats de petits cristaux arrondis et maclés d'environ $5 \mu \mathrm{m}$ de diamètre, la taille des cristaux variant entre 0,5 et $1 \mu \mathrm{m}$. Les zéolithes présentent un volume poreux accessible à l'azote de $0,240 \mathrm{~cm}^{3} \mathrm{~g}^{-1}$ (HMFI(40)) et $0,220 \mathrm{~cm}^{3} \mathrm{~g}^{-1}$ (HMFI(500)). Le nombre de sites acides de Brönsted déterminé par adsorption de pyridine suivie par infrarouge est de l'ordre de $300 \mu \mathrm{mol} \cdot \mathrm{g}^{-1}$ pour HMFI(40) (400 $\mu$ moles. $\mathrm{g}^{-1}$ théorique) et $30 \mu \mathrm{mol} \cdot \mathrm{g}^{-1}$ pour HMFI(500) $\left(33 \mu \mathrm{moles} \cdot \mathrm{g}^{-1}\right.$ théorique). Le nombre de sites acides très forts, capables de retenir la pyridine adsorbée à $450^{\circ} \mathrm{C}$, a été estimé à $50 \mu \mathrm{mol} \cdot \mathrm{g}^{-1}$ sur HMFI(40). Les sites acides présents sur silicalite sont relativement faibles puisque incapables de retenir la pyridine adsorbée à $250^{\circ} \mathrm{C}$.

\subsection{Adsorption du propène}

Les mesures d'adsorption du propène sur les zéolithes ont été réalisées sur une gamme de température allant de 25 à $450{ }^{\circ} \mathrm{C}$ à l'aide d'une microbalance de type Sartorius 4433. L'échantillon $(0,085 \mathrm{~g})$ est calciné sous air à $500^{\circ} \mathrm{C}$ pendant $3 \mathrm{~h}$. Le propène est introduit à $97 \mathrm{kPa}$ et la prise de masse de l'échantillon (notée \%m par la suite) suivie en fonction du temps. Après chaque expérience, afin de déterminer la quantité de matière non désorbable (coke et notée \%C par la suite), l'échantillon est traité sous vide (1 Pa) à la température de l'adsorption jusqu'à l'obtention d'une masse constante.

\subsection{Analyse de la phase adsorbée (coke)}

Pour analyser la phase adsorbée, plusieurs échantillons ont été préparés in situ en microbalance afin d'obtenir environ $1 \mathrm{~g}$ de zéolithe. Les composés ont été analysés en utilisant la technique développée au laboratoire pour l'analyse de coke [14]. La zéolithe est solubilisée à température ambiante dans de l'acide fluorhydrique à $40 \%$ afin de libérer les molécules organiques piégées dans les pores. Nous avions préalablement vérifié que cette technique n'affectait en rien la nature de ces composés organiques [15]. Les molécules de coke libérées des pores de la zéolithe sont solubilisées dans du 
chlorure de méthylène et analysées par CPG (colonne capillaire DB5) et par couplage chromatographie en phase gazeuse-spectrométrie de masse (CPG-SM).

\section{RÉSULTATS ET DISCUSSIONS}

\subsection{Adsorption du propène et formation de coke}

L'influence du temps sur l'adsorption (prise de masse :\%m) et sur la formation de coke $(\% \mathrm{C})$, obtenue après désorption sous vide de l'échantillon à la température d'adsorption, a été suivie sur HMFI(500) entre 25 et $450^{\circ} \mathrm{C}$. Excepté à $450{ }^{\circ} \mathrm{C}$, la prise de masse $(\% \mathrm{~m})$ est généralement rapide et une partie de la phase adsorbée peut être éliminée par traitement sous vide (fig. 1). La proportion de matière désorbée est variable d'une température à l'autre. Ainsi, après $70 \mathrm{~h}$ d'adsorption à $25^{\circ} \mathrm{C}, 75 \%$ de la phase adsorbée est éliminée alors que seulement $20 \%$ l'est à 100,150 et $350^{\circ} \mathrm{C}$. Aucun composé ne peut être éliminé sous vide à $450^{\circ} \mathrm{C}$. Paradoxalement, à $250^{\circ} \mathrm{C}$ une plus grande partie des composés retenus dans les pores de la zéolithe sont éliminables : $55 \%$ après $70 \mathrm{~h}$ et $40 \%$ après $120 \mathrm{~h}$. À cette température, la formation de coke (partie irréversiblement adsorbée) continue à augmenter avec le temps alors que pour les autres températures un palier est rapidement atteint.

Après $70 \mathrm{~h}$, la quantité adsorbée $(\% \mathrm{~m})$ sur la zéolithe HMFI(500) passe par un maximum entre 100 et $150^{\circ} \mathrm{C}$ puis diminue lorsque la température augmente (fig. 2). Pour les mêmes températures, la formation de coke $(\% \mathrm{C})$ passe également par un maximum, ce dernier étant plus prononcé que pour la prise de masse.

La formation de coke sur HMFI(40) passe également par un maximum centré à $100^{\circ} \mathrm{C}$. Quelle que soit la température, la production de coke est toujours plus importante sur ce dernier solide (fig. 3). Elle est 7,5 et 2,5 fois plus grande pour respectivement 25 et $100^{\circ} \mathrm{C}$ et environ 2 fois plus importante à plus haute température $\left(T \geq 150^{\circ} \mathrm{C}\right)$. Comme cela a déjà été montré pour d'autres structures zéolithiques, cette augmentation dans la formation de coke peut être directement attribuée aux différences d'acidité des deux zéolithes sans jamais cependant atteindre un facteur 10 correspondant à la différence de sites de Brönsted ( 300 et $30 \mu \mathrm{mol} / \mathrm{g}$ pour respectivement HMFI(40) et HMFI(500)). Toutefois, il est à noter que sur la zéolithe acide (HMFI(40)), la prise de masse (\%m) est toujours égale au dépôt de coke excepté à $250^{\circ} \mathrm{C}$ où celle-ci est, après $70 \mathrm{~h}$, environ 2,5 fois plus grande que le dépôt de coke.

\section{2 Évolution de la capacité d'adsorption à l'azote des zéolithes cokées}

Sur HMFI(500), la mesure du volume poreux accessible à l'azote a été réalisée sur tous les échantillons cokés. Pour certaines températures, la pression de propène a été modifiée afin d'obtenir des taux de coke variables. La figure 4 donne l'évolution du rapport $V / V o$ ( $V$ : volume poreux de la zéolithe cokée et $V o$ : volume poreux de la zéolithe fraîche) en fonction de la teneur en coke et ceci pour les différentes températures d'adsorption. La perte de capacité d'adsorption diminue de façon quasi linéaire avec le taux de coke. Entre 25 et $250^{\circ} \mathrm{C}$, l'effet de la température n'a que peu d'influence sur la perte d'adsorption. Ainsi par extrapolation, $9 \%$ de coke sont nécessaires pour bloquer totalement la porosité de la zéolithe. En revanche, pour des températures plus élevées $\left(350-450{ }^{\circ} \mathrm{C}\right), 3 \%$ de coke semblent suffisants pour provoquer un bouchage de pores.

Pour une température donnée $\left(T=250{ }^{\circ} \mathrm{C}\right)$, la capacité d'adsorption à l'azote sur HMFI(500) diminue plus rapidement avec le dépôt de coke que celle mesurée sur HMFI(40) (fig. 5). En effet, si on observe des droites parallèles, environ $12 \%$ de coke sont nécessaires pour bloquer l'accès de l'azote à la microporosité de la zéolithe HMFI(40) contre $9 \%$ sur $\operatorname{HMFI}(500)$.

\subsection{Analyse de la phase adsorbée}

La phase irréversiblement adsorbée désignée sous le terme coke a été analysée sur HMFI(40) et HMFI(500) après adsorption du propène à $25,150,250$ et $350^{\circ} \mathrm{C}$. Les conditions opératoires ont été ajustées de façon à obtenir, pour une même température, des quantités de coke voisines.

Sur HMFI(40), quelle que soit la température d'adsorption les molécules constituant le coke sont toujours totalement solubles dans le chlorure de méthylène après solubilisation de la zéolithe cokée dans l'acide fluorhydrique. Par contre sur HMFI(500), si les molécules de coke sont totalement solubles dans le chlorure de méthylène entre 25 et $250{ }^{\circ} \mathrm{C}$, environ $70 \%$ reste insolubles à $350^{\circ} \mathrm{C}$ malgré un pourcentage de coke relativement faible $(2,5 \%)$ obtenu après $70 \mathrm{~h}$ d'adsorption. L'analyse par couplage CPG/SM des extraits de coke permet de définir leurs compositions (tableau 1).

De 25 à $150^{\circ} \mathrm{C}$, le propène est transformé en oligomères sur les deux zéolithes. Toutefois, quelques différences peuvent être notées. À $25{ }^{\circ} \mathrm{C}$, les oligomères sont constitués d'alcènes ayant un nombre d'atomes de carbone multiple de 3 et pouvant varier de 9 à 24 atomes de carbone sur HMFI(40) et de 9 à 15 sur HMFI(500). Des traces d'oligomères non multiples de 3 carbones sont également détectées sur HMFI(40) pour des temps d'adsorption relativement longs $(t=70 \mathrm{~h} ; \% \mathrm{C}=15 \%)$.

À $150^{\circ} \mathrm{C}$, les oligomères présents sur les deux zéolithes possèdent un nombre d'atomes de carbone non multiple de 3 et compris entre 12 et 34 . Le degré d'insaturation de ces composés varie d'une zéolithe à l'autre: ils sont très insaturés sur HMFI(40) et beaucoup moins sur HMFI(500) (tableau 1). 

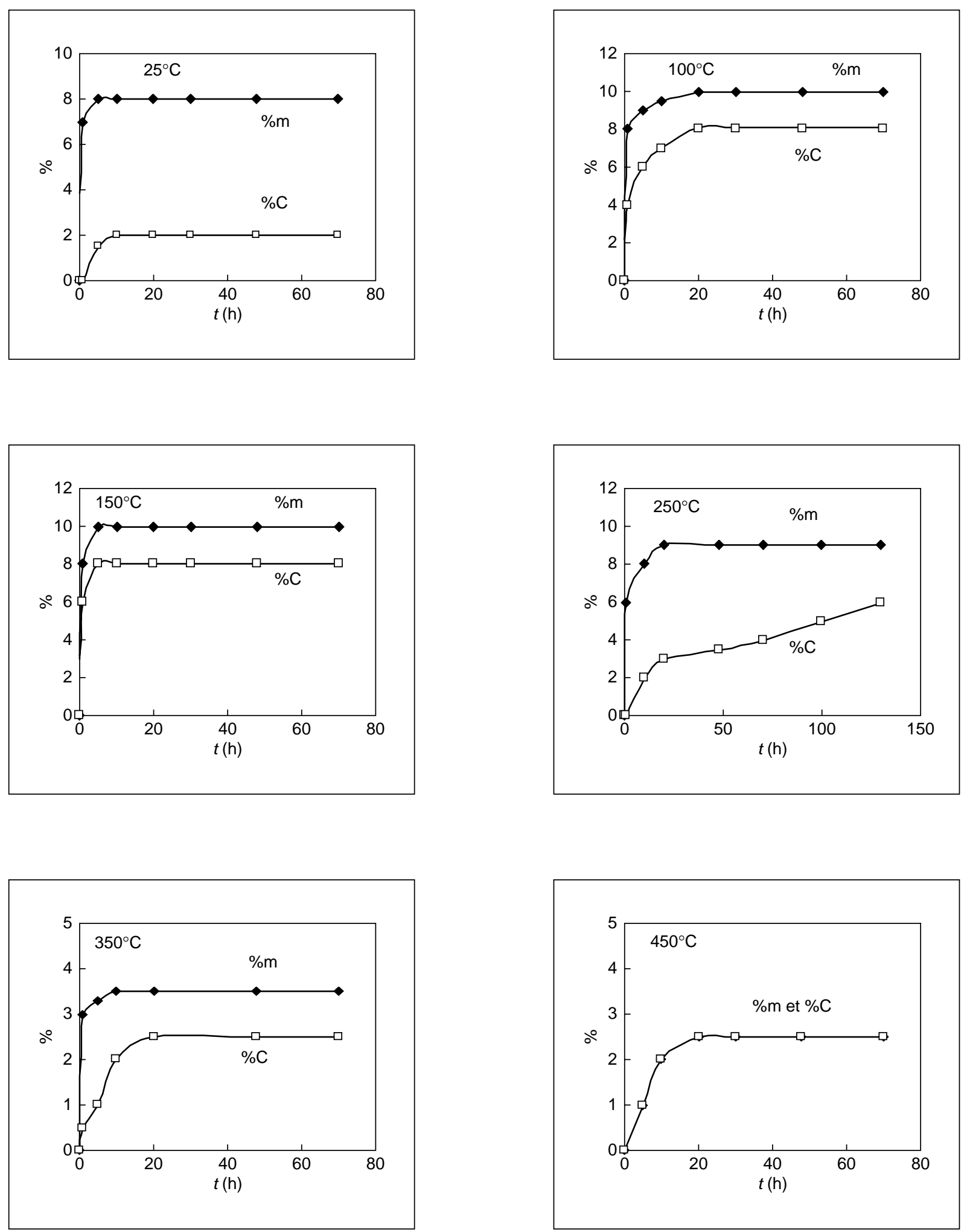

Figure 1

Prise de masse $(\% \mathrm{~m})$ et pourcentage de coke $(\% \mathrm{C})$ sur HMFI(500) en fonction du temps d'adsorption. Conditions opératoires : 25 à $450{ }^{\circ} \mathrm{C}$, $\mathrm{P}_{\text {propène }}=97 \mathrm{kPa}$.

Increase in weight $(\% \mathrm{~m})$ and coke content $(\% \mathrm{C})$ on $\mathrm{HMFI}(500)$ vs operating time. Operating conditions: 25 to $450^{\circ} \mathrm{C}, P_{\text {propene }}=97 \mathrm{kPa}$. 


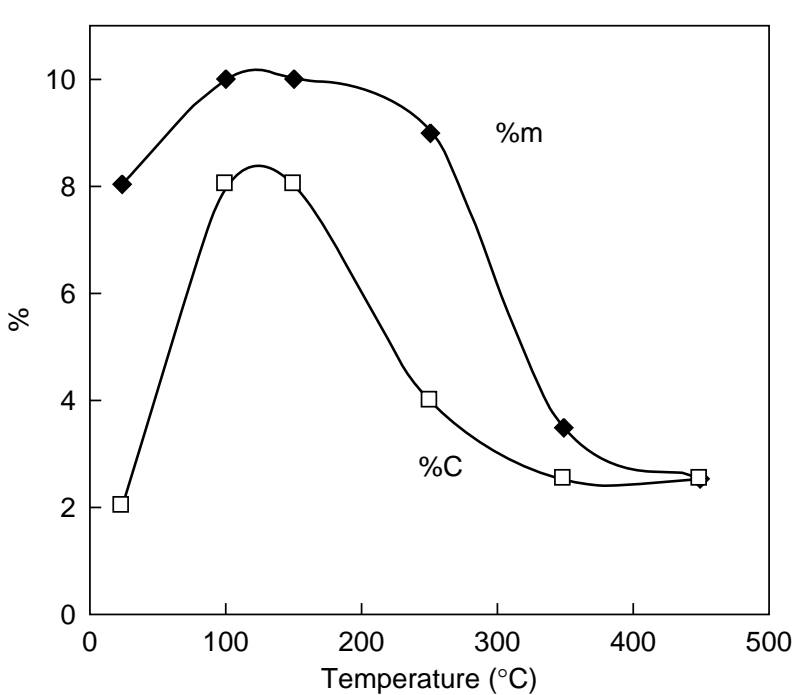

Figure 2

Prise de masse $(\% \mathrm{~m})$ et pourcentage de coke $(\% \mathrm{C})$ pris après $70 \mathrm{~h}$ sur HMFI(500) en fonction de la température d'adsorption. Increase in weight (\%) and coke content (\% C) taken after $70 \mathrm{~h}$ on $\operatorname{HMFI}(500)$ as a function of the adsorption temperature.

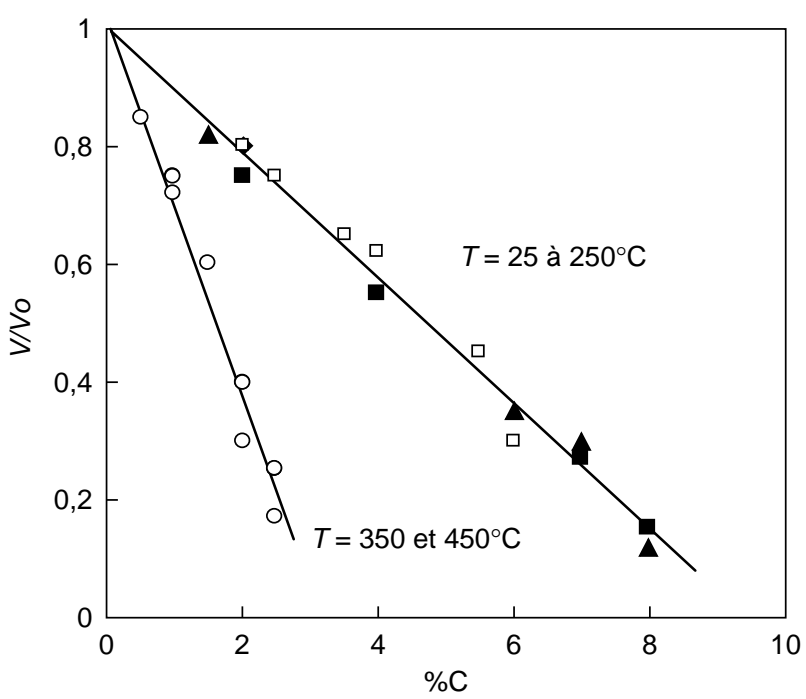

Figure 4

Variation de la capacité d'adsorption à l'azote sur HMFI(500) en fonction du pourcentage de carbone déposé entre 25 et $450{ }^{\circ} \mathrm{C}$.

Capacity for nitrogen adsorption of a HMFI(500) as a function of the amount of coke deposited from 25 to $450{ }^{\circ} \mathrm{C}$.

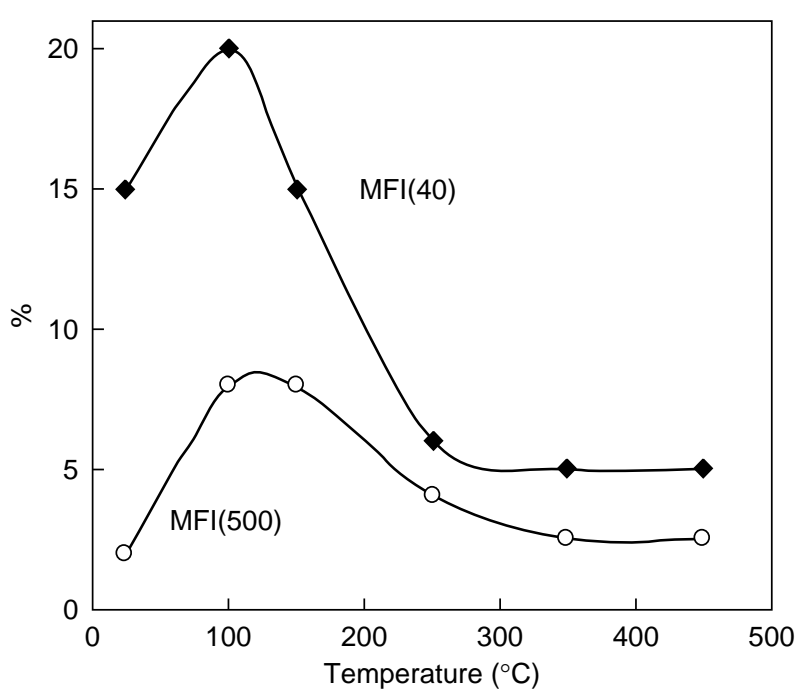

Figure 3

Pourcentage de coke $(\% \mathrm{C})$ pris après $70 \mathrm{~h}$ sur HMFI(40) et HMFI(500) en fonction de la température d'adsorption.

Coke content $(\% \mathrm{C})$ taken after $70 \mathrm{~h}$ on HMFI(40) and HMFI(500) as a function of the adsorption temperature.

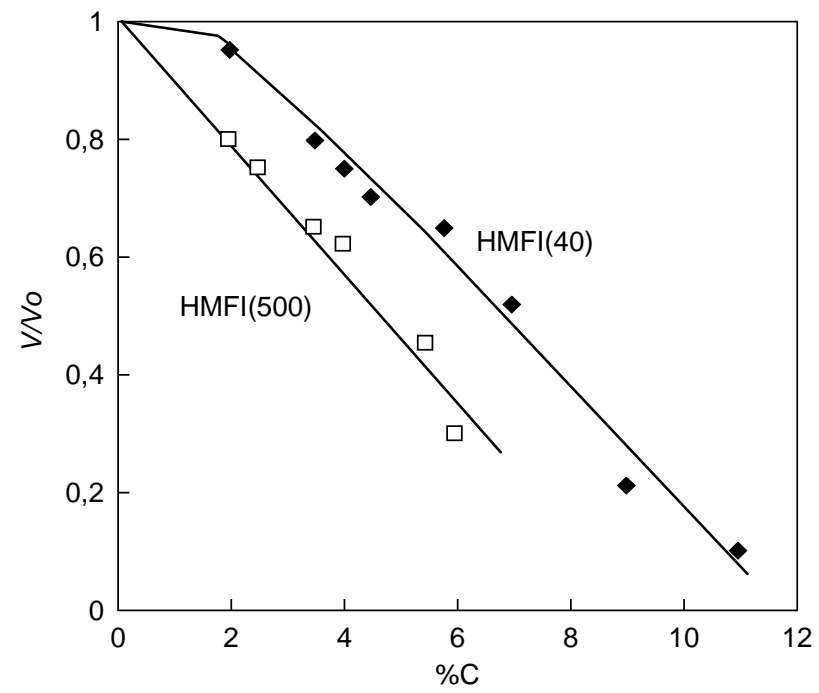

Figure 5

Variation de la capacité d'adsorption à l'azote sur HMFI(40) et $\operatorname{HMFI}(500)$ en fonction du pourcentage de carbone déposé à $250{ }^{\circ} \mathrm{C}$.

Capacity for nitrogen adsorption of a HMFI(40) and HMFI(500) as a function of the amount of coke deposited at $250^{\circ} \mathrm{C}$. 
TABLEAU 1

Constituants majoritaires du coke après adsorption du propène sur HMFI(40) et HMFI(500)

Main compounds of coke formed from propene over HMFI(40) and HMFI(500)

\begin{tabular}{|c|c|c|}
\hline Température & $\operatorname{HMFI}(40)$ & $\operatorname{HMFI}(500)$ \\
\hline $25^{\circ} \mathrm{C}$ & $\begin{array}{l}\% \mathrm{C}=5 \\
\mathrm{C}_{n} \mathrm{H}_{2 \mathrm{n}} \\
\mathrm{C}_{9} \mathrm{H}_{18}, \mathrm{C}_{12} \mathrm{H}_{24},{ }^{*} \mathrm{C}_{15} \mathrm{H}_{30}, \\
\mathrm{C}_{18} \mathrm{H}_{36}, \mathrm{C}_{21} \mathrm{H}_{42}, \mathrm{C}_{24} \mathrm{H}_{48}\end{array}$ & $\begin{array}{l}\% \mathrm{C}=2 \\
\mathrm{C}_{n} \mathrm{H}_{2 \mathrm{n}} \\
\mathrm{C}_{9} \mathrm{H}_{18},{ }^{*} \mathrm{C}_{12} \mathrm{H}_{24}, \mathrm{C}_{15} \mathrm{H}_{30}\end{array}$ \\
\hline $150^{\circ} \mathrm{C}$ & $\begin{array}{l}\% \mathrm{C}=9 \\
\mathrm{C}_{n} \mathrm{H}_{2 n},{ }^{*} \mathrm{C}_{n} \mathrm{H}_{2 n+2}, \mathrm{C}_{n} \mathrm{H}_{2 n-2}, \\
\mathrm{C}_{n} \mathrm{H}_{2 n-4}\left(\mathrm{C}_{12} \text { à } \mathrm{C}_{34}\right)\end{array}$ & $\begin{array}{l}\% \mathrm{C}=8 \\
\mathrm{C}_{\mathrm{n}} \mathrm{H}_{2 \mathrm{n}} \\
\left(\mathrm{C}_{12} \text { à } \mathrm{C}_{30}\right)\end{array}$ \\
\hline $250^{\circ} \mathrm{C}$ & $\left(\mathrm{CH}_{2}\right)_{\mathrm{x}}-\mathrm{CH}_{3}$ & $\begin{array}{l}\% \mathrm{C}=6 \\
{ }^{*} \mathrm{C}_{n} \mathrm{H}_{2 \mathrm{n}+2}, \mathrm{C}_{n} \mathrm{H}_{2 \mathrm{n}+2}, \mathrm{C}_{\mathrm{n}} \mathrm{H}_{2 \mathrm{n}-2} \\
\left(\mathrm{C}_{12} \mathrm{à} \mathrm{C}_{30}\right)\end{array}$ \\
\hline $350^{\circ} \mathrm{C}$ & $\% \mathrm{C=5}$ & $\begin{array}{l}\text { - }\left(\mathrm{CH}_{3}\right)_{x} \\
+\quad \text { * polyaromatiques }\end{array}$ \\
\hline
\end{tabular}

* Composés majoritaires

À $250^{\circ} \mathrm{C}$, des alkylbenzènes et aklylnaphtalènes à longues chaînes sont détectés sur HMFI(40). Sur HMFI(500), une coexistence entre alkylbenzènes et oligomères est observée (tableau 1).

À $350^{\circ} \mathrm{C}$, le coke soluble dans le chlorure de méthylène est constitué, sur les deux zéolithes, de méthylnaphtalène, antracène, phénanthrène et pyrène. Par contre sur HMFI(500), une forte proportion de composés aromatiques insolubles dans les solvants est mise en évidence (tableau 1).

\section{DISCUSSION}

\subsection{Mode de formation de coke}

Le propène peut être adsorbé et transformé en divers composés sur les zéolithes HMFI. Une partie de ces composés peut être éliminée des pores par un simple traitement sous vide à la température de l'adsorption, l'autre partie restant bloquée dans les pores (coke). 
À basse température $\left(T \leq 150^{\circ} \mathrm{C}\right)$ le propène est transformé sur les deux zéolithes en oligomères retenus dans les pores de la zéolithe puisque non désorbables sous vide et ne pouvant être extraits directement par un solvant. En accord avec la littérature $[16,17]$, les oligomères formés dans les pores de la zéolithe HMFI sont peu ramifiés. Le rapport $\mathrm{CH}_{3} /\left(\mathrm{CH}_{2}+\mathrm{CH}\right)$ mesuré par HRMN est voisin de 0,25 , ce qui correspond à une moyenne de 1 à 2 branchements pour une gamme de produits possédant de 9 à 34 atomes de carbone.

À $25^{\circ} \mathrm{C}$, le propène subit une simple réaction d'oligomérisation qui conduit à la formation d'alcènes possédant un nombre d'atomes de carbone multiple de 3 (étape 1).

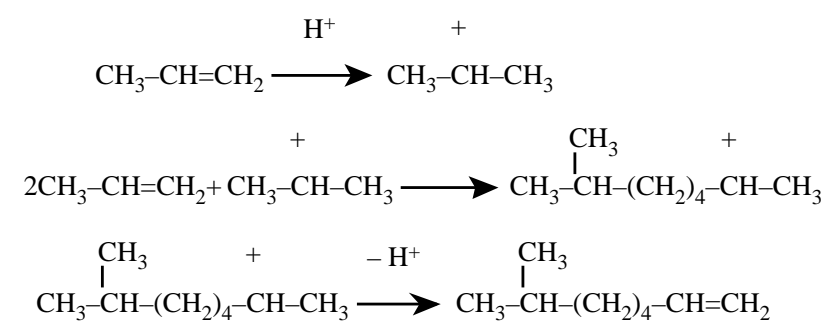

(étape 1)

La réaction d'oligomérisation se poursuit bien plus loin sur HMFI(40) puisque des molécules à 24 atomes de carbone ont pu être détectées. Ceci est certainement dû à la plus forte acidité de cette zéolithe. Ces alcènes sont retenus dans les pores de la zéolithe à cause de leur faible volatilité mais aussi certainement en raison de leur encombrement stérique. Toutefois, les alcènes majoritairement retenus sont les dodécènes sur HMFI(500) et les pentadécènes sur HMFI(40). Les nonènes sont minoritaires dans tous les cas, certainement à cause d'une désorption partielle des pores de la zéolithe. On peut imaginer également que des héxènes peuvent être formés mais non retenus en raison de leur faible volatilité. D'ailleurs, des résultats récents indiquent que des méthyl-pentanes peuvent, à basse température, pénétrer dans les pores de la zéolithe HMFI avec une possibilité de désorption [6].

Entre 100 et $150^{\circ} \mathrm{C}$, des oligomères possédant un nombre d'atomes de carbone non multiple de 3 sont formés et retenus dans les pores des zéolithes. Ces molécules ne sont pas formées à partir de simples réactions d'oligomérisation entre molécules de propène, mais à partir de réactions plus complexes faisant intervenir plusieurs étapes :

- oligomérisation du propène sur sites acides (étape 1);

- craquage de ces oligomères sur les sites acides (étape 2);

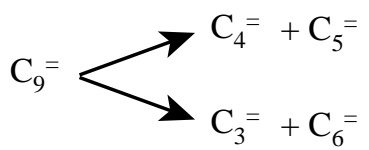

- réactions des produits de craquage avec le propène, entre eux ou avec les oligomères déjà formés pour conduire à des alcènes à longues chaînes (étape 3 );

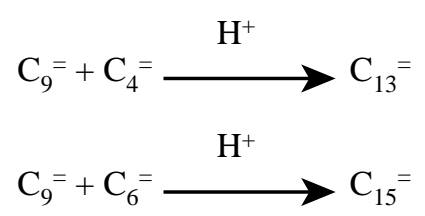

(étape 3)

- transfert d'hydrogène entre oligomères insaturés pour conduire à des alcanes et des composés plus insaturés (étape 4).

$$
2 \mathrm{C}_{\mathrm{n}} \mathrm{H}_{2 \mathrm{n}} \longrightarrow \mathrm{C}_{\mathrm{n}} \mathrm{H}_{2 \mathrm{n}+2}+\mathrm{C}_{\mathrm{n}} \mathrm{H}_{2 \mathrm{n}-2} \quad \text { (étape 4) }
$$

Sur la zéolithe la moins acide (HMFI(500)), les réactions de transfert d'hydrogène sont inexistantes et les produits formés sont des alcènes possédant de 12 à 30 atomes de carbone. Sur la zéolithe la plus acide, les réactions de transfert d'hydrogène sont favorisées et conduisent à la formation d'alcanes et de composés très insaturés $\left(\mathrm{C}_{\mathrm{n}} \mathrm{H}_{2 \mathrm{n}-4}\right)$. Ces résultats montrent clairement que les sites acides forts présents sur HMFI(40) participent aux réactions de transfert d'hydrogène et que cette réaction bimoléculaire est bien favorisée sur une zéolithe à densité de site acide élevée $[18,19]$ ce qui se traduit également par une formation de coke plus importante sur HMFI(40).

La formation de tous ces composés est favorisée entre 100 et $150^{\circ} \mathrm{C}$ et leur forte rétention dans les pores des zéolithes HMFI (fig. 3) est essentiellement due à leur faible volatilité.

À $250^{\circ} \mathrm{C}$, la tendance au cokage des deux zéolithes est beaucoup plus faible qu'à $150^{\circ} \mathrm{C}$, ceci pouvant être lié à la compétition entre rétention et diffusion des produits formés. Ceci est clairement montré figure 2 sur $\operatorname{MFI}(500)$ où la prise de masse est environ 2,3 fois plus importante que le dépôt de carbone. Ainsi, des molécules volatiles, résultant certainement du craquage des oligomères et/ou des aromatiques à longues chaînes, peuvent facilement être désorbées sous vide. Les molécules formées dans les pores des zéolithes à cette température sont relativement différentes d'une zéolithe à l'autre. Ainsi pour une teneur en carbone de $6 \%$, seuls les composés aromatiques sont présents sur la zéolithe la plus acide (HMFI(40)) alors que sur la zéolithe HMFI(500), des oligomères sont encore détectés, confirmant ainsi que les réactions de cyclisation, de transfert d'hydrogène et d'alkylation sont plus limitées lorsque le nombre et la force des sites acides de la zéolithe sont faibles. En effet, les aromatiques à longues chaînes retenus dans les pores des zéolithes sont formés par diverses réactions successives : oligomérisation, cyclisation, transfert d'hydrogène et alkylation des aromatiques par les oligomères. Tous ces composés restent piégés dans la microporosité des zéolithes à cause de leurs faibles volatilités.

À $350^{\circ} \mathrm{C}$, les composés constituant le coke soluble dans le dichlorométhane sont peu différents d'une zéolithe à l'autre (tableau 1). Ils sont formés à partir des composés aromatiques plus simples détectés à $250^{\circ} \mathrm{C}$ et résultent de réactions 
successives de cyclisation et transfert d'hydrogène. Ces composés restent piégés dans les pores des zéolithes en raison de leur encombrement stérique et pourraient être plus particulièrement localisés à l'intersection des canaux de la zéolithe HMFI, où l'espace disponible est le plus important (environ 8,5 ̊). La possibilité d'une localisation de la molécule de pyrène à l'intersection des canaux de la zéolithe MFI est d'ailleurs confirmée par modélisation moléculaire [16]. Paradoxalement, les constituants du coke à $350{ }^{\circ} \mathrm{C}$ sont beaucoup plus polyaromatiques sur la zéolithe la moins acide (présence majoritaire de composés insolubles dans le dichlorométhane). Ces résultats sont en accord avec ceux déjà décrits sur zéolithes HY [19] et HEMT [20] possédant des rapports $\mathrm{Si} / \mathrm{Al}$ élevés. Ce phénomène a été attribué à des réactions de condensation entre les molécules de coke solubles à l'intérieur des pores des zéolithes :
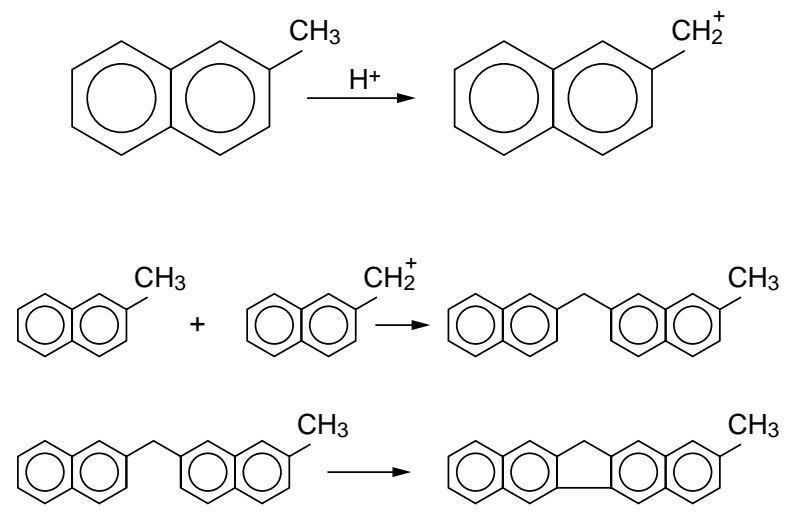

Ce mode de formation de coke serait favorisé lorsque le nombre de sites acides de la zéolithe est faible et le temps de réaction important [19]. De plus, la formation de ces composés très polyaromatiques est également favorisée lorsque le catalyseur désactivé est laissé un certain temps à la température de réaction en absence de réactif et sous gaz inerte [21].

\subsection{Localisation des molécules de coke}

Malgré le nombre de sites acides peu important et la faible acidité de la zéolithe HMFI(500), le propène conduit, à $25^{\circ} \mathrm{C}$, à la formation d'oligomères dans les pores de la zéolithe. Une estimation du volume occupé par ces molécules a été calculée à partir de leur composition et de leur densité en phase liquide (densité moyenne de $0,76 \mathrm{~g} / \mathrm{cm}^{3}, \mathrm{~d}_{\mathrm{C} 12 \mathrm{H} 24}$ $\left.=0,7584 \mathrm{~g} / \mathrm{cm}^{3}\right)$. À saturation, le volume occupé par ces oligomères serait donc de $0,026 \mathrm{~cm}^{3} / \mathrm{g}$ soit un volume 8,5 fois plus faible que celui de la zéolithe. Sur HMFI(40), le dépôt de coke est 7,5 fois plus élevé, ce qui correspond à un volume occupé par les oligomères d'environ $0,19 \mathrm{~cm}^{3} / \mathrm{g}$, soit $80 \% \mathrm{du}$ volume de la zéolithe $\left(0,240 \mathrm{~cm}^{3} / \mathrm{g}\right)$. Le nombre de molécules de coke par gramme de zéolithe $\left(\mathrm{N}_{\mathrm{k}}\right)$, estimé à partir de la masse molaire des composés prédominants, est égal à $0,7110^{20}$ et $4,310^{20}$ pour respectivement $\operatorname{HMFI}(500)$ et
HMFI(40). Quelle que soit la zéolithe, $\mathrm{N}_{\mathrm{k}}$ est de 2 à 4 fois plus grand que le nombre de sites acides des zéolithes. Ce résultat montre que si les sites acides initient la réaction d'oligomérisation, les oligomères formés peuvent ensuite rester piégés dans les pores des zéolithes sans être adsorbés sur des sites acides mais certainement en restant bloqués entre deux autres molécules. Ceci est également vrai à 100 et $150^{\circ} \mathrm{C}$ où le nombre de molécules de coke $\mathrm{N}_{\mathrm{k}}$ est toujours supérieur au nombre de sites acides des zéolithes. De plus à $100^{\circ} \mathrm{C}, 50 \%$ de la porosité de HMFI(500) est occupée par les oligomères et la totalité du volume poreux de HMFI(40) est occupé.

À $250^{\circ} \mathrm{C}, 12 \%$ de coke semblent nécessaires pour bloquer totalement la porosité de la zéolithe HMFI(40) alors que $9 \%$ sont suffisants pour rendre la porosité de HMFI(500) inaccessible à l'azote (fig. 5). Ainsi, 45 et $50 \%$ du volume poreux des zéolithes HMFI(500) et HMFI(40) sont occupés par des molécules de coke (alkylaromariques et oligomères). Cette plus forte désactivation observée sur HMFI(500) peut être attribuée à la présence résiduelle d'oligomères dans ses pores. Cette zéolithe moins acide et possédant des sites beaucoup plus faibles que son homologue HMFI(40) n'est pas capable à $250^{\circ} \mathrm{C}$ de transformer et /ou craquer tous les oligomères formés. La présence de ces derniers dans les canaux de la zéolithe provoque donc un bloquage de pores plus prononcé que sur HMFI(40) où seuls des composés aromatiques sont présents.

À 350 et $450^{\circ} \mathrm{C}$, des molécules très polyaromatiques sont formées sur HMFI(500) et $3 \%$ de coke sont suffisants pour bloquer totalement la porosité de la zéolithe (fig. 4). Ceci indique un processus de bloquage de pores prononcé résultant de la formation de molécules de coke très volumineuses et polyaromatiques localisées préférentiellement sur la surface externe des cristallites de zéolithe [14].

\section{CONCLUSION}

La formation de coke ainsi que son effet désactivant ont été étudiés sur silicalite (HMFI(500)) et sur zéolithe MFI plus acide (HMFI(40)) pour diverses températures d'adsorption. Malgré la faible acidité de la silicalite, aussi bien en nombre de sites qu'en force, le propène conduit à $25^{\circ} \mathrm{C}$ à la formation d'oligomères bloqués dans les pores de la zéolithe. Ces composés sont formés en raison des quelques sites acides présents dans les canaux de la zéolithe. Ils résultent de réactions successives d'oligomérisation avec formation d'oléfines ayant 9, 12 et 15 atomes de carbone. Sur HMFI(40), la formation de ces composés est plus rapide et les oligomères formés sont plus volumineux $\left(\mathrm{C}_{9}\right.$ à $\left.\mathrm{C}_{24}\right)$. La quantité maximale de coke obtenue sur silicalite à partir du propène se situe autour de $100-150^{\circ} \mathrm{C}$. Pour ces températures, des oligomères sont encore formés mais des réactions d'oligomérisation/ craquage apparaissent et le transfert d'hydrogène est favorisé sur la zéolithe la plus acide. Sur cette dernière, à $100^{\circ} \mathrm{C}$, 
$20 \%$ de coke peuvent être déposés et dans ces conditions, les molécules formées occupent la totalité de la porosité de la zéolithe. De plus, quelles que soient la zéolithe et la température d'adsorption $\left(25\right.$ à $\left.150{ }^{\circ} \mathrm{C}\right)$, le nombre de molécules de coke est toujours supérieur au nombre de sites acides présents dans la zéolithe. Ceci indique que les sites protoniques servent uniquement à initier la réaction d'oligomérisation, les molécules formées étant ensuite piégées dans les pores des zéolithes sans être adsorbées sur les sites acides. Entre 250 et $450{ }^{\circ} \mathrm{C}$, des composés aromatiques sont formés et sont localisés soit dans les canaux (alkylbenzènes et alkylnaphtalènes) soit à l'intersection des canaux (dérivés du pyrène). Sur HMFI(500), des composés polyaromatiques sont mis en évidence à partir de $350{ }^{\circ} \mathrm{C}$, leur formation étant favorisée lorsque le nombre de sites acides est faible et le temps d'adsorption très grand. La présence de ces molécules provoque rapidement un bloquage total de la porosité de la zéolithe.

\section{RÉFÉREN CES}

1 Belloum, M., Travers, Ch. et Bournonville, J.P. (1991) Isomérisation des paraffines de $\mathrm{C}_{4}$ à $\mathrm{C}_{7}$ sur catalyseurs zéolithiques. Revue de l'Institut français du pétrole, 46, 1, 89107.

2 Jullian, S., Mank, L. et Minkkinen, A. (1993) Fr. Patent, 2.679.245, US Patent, 5.233.120.

3 Célio, L., Cavalcante, Jr., et Ruthven, D.M. (1995) Adsorption of Branched and Cyclic Paraffins in Silicalite. 1. Equilibrium. Ind. Eng. Chem. Res, 34, 177-184.

4 Célio, L., Cavalcante, Jr., et Ruthven, D.M. (1995) Adsorption of Branched and Cyclic Paraffins in Silicalite. 2. Kinetics. Ind. Eng. Chem. Res, 34, 185-191.

5 Jolimaitre, E., Tayakout-Fayolle, M., Jallut, C. et Ragil, K. (2001) Determination of Mass Transfer and Thermodynamic Properties of Branched Paraffins in Silicalite by Inverse Chromatography Technique. Ind. Eng. Chem. Res, 40, 914926.

6 Gener, I., Rigoreau, J., Joly, G., Renaud, A. et Mignard, S. (2002) Adsorption of Linear and Branched Paraffins in Silicalite: Thermodynamic and Kinetic Study. Stud. Surf. Sci. Catal., 142, 1679-1684.

7 Magnoux, P., Boucheffa, Y., Guisnet, M., Joly, G., et Jullian, S. (1998) Evidence for Isopentane Adsorption over 5A Zeolite. 6th Fundamentals of Adsorption, 135-140.

8 Magnoux, P., Boucheffa, Y., Guisnet, M., Joly, G., et Jullian, S. (2000) Mise en évidence de l'adsorption de l'isopentane dans les pores de la zéolithe 5A. Oil \& Gas Science and Technology - Rev. IFP, 55, 3, 307-314.
9 Misk, M., Joly, G., Magnoux, P., Jullian, S. et Guisnet, M. (1996) Coking, Aging and Regeneration of Zeolites: XVII. Composition and Location of Carbonaceous Compounds Resulting from Isobutene and Propene Transformation on a 5A Zeolite. Zeolites, 16, 265-270.

10 Thomazeau, C., Cartraud, P., Magnoux, P., Jullian, S. et Guisnet, M. (1996) Formation from Propene of Carbonaceous Compounds Responsible for the Deactivation of a 5A Adsorbent. Removal through Pyrolysis and Oxidative Treatment. Microporous Materials, 5, 337-345.

11 Magnoux, P., Misk, M., Joly, G., Jullian, S. et Guisnet, M. (1997) Composition, Location, Modes of Formation and of Removal of Coke Deposited on a 5A Adsorbent. Stud. Surf. Sci. Catal., 105, 1835-1858.

12 Boucheffa, Y., Thomazeau, C., Cartraud, P., Magnoux, P., Guisnet, M. et Jullian, S. (1997) Formation of Carbonaceous Compounds from Propene and Isobutene over a 5A Zeolite. Influence of Temperature on their Compositions and Locations. Ind. Eng. Chem. Res., 36, 3198-3204.

13 Misk, M., Joly, G., Magnoux, P., Guisnet, M., et Jullian, S. (2000) Formation of Coke from Propene over 5A Adsorbents-Influence of the Binder on the Coke Composition, Location and Removal. Microporous and Mesoporous Materials, 40, 197-204.

14 Guisnet, M., et Magnoux, P. (1989) Coking and Deactivation of Zeolites. Influence of the Pore Structure. Appl. Catal., 54, 1-27.

15 Magnoux, P., Roger, P., Canaff, C., Fouché, V., Gnep, N.S. et Guisnet, M. (1987) New Technique for the Characterization of Carbonaceous Compounds Responsible for Zeolite Deactivation. Stud. Surf. Sci. Catal., 34, 317-330.

16 Guisnet, M., et Magnoux, P. (2001) Organic Chemistry of Coke Formation. Appl. Catal., 54, 1-27.

17 Ghosh, A.K. et Kydd, A.R. (1986) A Fourier-Transformation Infrared Study of Propene Reactions on Acidic Zeolites. J. Catal., 100, 185-195.

18 Cheng, W.C., et Rajagopalan, K. (1989) Conversion of Cyclohexene over Y-Zeolites: A Model Reaction for Hydrogen Transfer. J. Catal., 119, 354-358.

19 Moljord, K., Magnoux, P. et Guisnet, M. (1995) Influence of the Composition of HY Zeolites on the Mode of Formation of Coke from Propene at $450^{\circ} \mathrm{C}$. Appl. Catal. A: General, 122, 21-32.

20 Doka Nassionou, G.A., Magnoux, P. et Guisnet, M. (1998) Comparative Study of Coking and Regeneration of HEMT and HFAU Zeolites. Microporous and Mesopourous Materials, 22, 389-398.

21 Magnoux, P., Cerqueira, H.S., et Guisnet M. (2002) Evolution of Coke Composition During Ageing under Nitrogen. Appl. Catal. A: General, 235, 93-99.

Manuscrit définitif reçu en février 2003 